\title{
Anomaly Driven Cosmology: Big Boost Scenario and AdS/CFT Correspondence
}

\author{
A.O.Barvinsky ${ }^{1}$, C.Deffayet ${ }^{2}$ and A.Yu.Kamenshchik ${ }^{3,4}$
}

\author{
${ }^{1}$ Theory Department, Lebedev Physics Institute, Leninsky Prospect 53, Moscow 119991, Russia \\ ${ }^{2}$ APC UMR 7164 (CNRS, Univ. Paris 7, CEA, Obs. de Paris), 12 rue Alice Domon et Léonie \\ Duquet, 75205 Paris Cedex 13, France \\ ${ }^{3}$ Dipartimento di Fisica and INFN, via Irnerio 46, 40126 Bologna, Italy \\ ${ }^{4}$ L.D.Landau Institute for Theoretical Physcis of Russian Academy of Sciences, Kosygin str. 2, 119334 \\ Moscow, Russia
}

\begin{abstract}
We consider the cosmological evolution in a recently suggested new model of quantum initial conditions for the Universe. The effective Friedmann equation incorporates the effect of the conformal anomaly of quantum fields and, interestingly, shows that their vacuum Casimir energy is completely screened and does not gravitate. The cosmological evolution also features a new mechanism for a cosmological acceleration stage. This stage is followed by a big boost singularity - a rapid growth up to infinity of the scale factor acceleration parameter. We also briefly discuss the relation between our model, the AdS/CFT correspondence and RS and DGP braneworlds.
\end{abstract}

\section{Introduction}

Conformally invariant fields play a very important role in physics. In particular, the success of string theory is largely due to the fact that local conformal invariance provides us with the means of exactly solving the underlying $2 \mathrm{D}$ problem. Conformal invariance is pertinent not only to deal with the 2D dynamics on the string world sheet, but also manifests itself in field theoretical implications of string theory like the AdS/CFT correspondence suggesting new calculational methods to describe a strongly coupled regime. On the other hand, conformally invariant fields have important implications in cosmology of the early Universe. Cosmological evolution driven by the conformal anomaly of conformally invariant fields [1, 2] represented perhaps first examples of self-consistent inflationary models. These earlier works disregarded the formulation of initial conditions which later were considered in the form of the no-boundary proposal [3] within the conformal anomaly context and, more recently, using the AdS/CFT correspondence [4].

Some of these ideas were recently generalized in a new model of quantum initial conditions for the Universe [5]. In this model, one considers a (possibly large) number of conformal fields as the initial matter content of the Universe, also endowed with a cosmological constant, and allows the possibility that the initial state of the Universe be a mixed state, rather that a pure state as in the Harle-Hawking proposal. Using the conformal invariance, it is then possible in this model to compute the statistical sum in quantum gravity of spatially closed cosmologies [5. Indeed, the initial state is represented by the microcanonical density matrix [6] whose statistical sum can be calculated within the $1 / N_{\text {cdf }}$-expansion in the number, $N_{\text {cdf }}$, of conformally invariant quantum fields under the assumption that they outnumber all other degrees of freedom. This statistical sum is dominated by the set of the quasi-thermal cosmological instantons. A first very interesting outcome of this calculation is the fact that its consistency requires the effective cosmological constant of the early Universe to belong to a finite range, strictly bounded from above and from below [5]. It also shows that the vacuum Hartle-Hawking instantons are excluded from the initial conditions, having infinite positive Euclidean gravitational effective action [5] due to the contribution of the conformal anomaly. 
Here we are going to analyze the cosmological evolution in this model with the initial conditions set by the instantons of [5]. In particular, we will derive the modified Friedmann equation incorporating the effect of conformal anomaly at late radiation and matter domination stages. As will be shown, this equation has several interesting properties. First it shows that the vacuum (Casimir) part of the energy density is "degravitated" via the effect of the conformal anomaly. Namely the Casimir energy does not weigh. Second, we will show that this equation, together with the recovery of the general relativistic behavior, can feature a stage of cosmological acceleration followed by what we call a big boost singularity. At this singularity the scale factor acceleration grows in finite proper time up to infinity with a finite limiting value of the Hubble factor, when the Universe again enters a quantum phase demanding for its description a UV completion of the low-energy semiclassical theory. Finally we discuss the possibility of realizing this scenario within the AdS/CFT and braneworld setups, in particular when the conformal anomaly and its effective action are induced on $4 \mathrm{D}$ boundary/brane from the type IIB supergravity theory in the $5 \mathrm{D}$ bulk. We also comment on the relation between our model and the DGP setup.

\section{Cosmological initial conditions generated by the conformal anomaly}

The statistical sum for the microcanonical ensemble in spatially closed cosmology ( $S^{3}$-topology of spatial sections) was shown to be represented by the path integral over the periodic scale factor $a(\tau)$ and lapse function $N(\tau)$ of the minisuperspace metric

$$
d s^{2}=N^{2}(\tau) d \tau^{2}+a^{2}(\tau) d^{2} \Omega^{(3)}
$$

on the toroidal spacetime of $S^{1} \times S^{3}$ topology [6]

$$
e^{-\Gamma}=\int_{\text {periodic }} D[a, N] e^{-\Gamma_{E}[a, N]} .
$$

Here $\Gamma_{E}[a, N]$ is the Euclidean effective action of all inhomogeneous "matter" fields which include also metric perturbations on minisuperspace background of (2.1).

Under the assumption that the system is dominated by free matter fields conformally coupled to gravity this action is exactly calculable by the conformal transform from (2.1) to static Einstein metric with $a=$ const [5]. In units of the Planck mass $m_{P}=(3 \pi / 4 G)^{1 / 2}$ it reads

$$
\begin{aligned}
& \Gamma_{E}[a, N]=m_{P}^{2} \int d \tau N\left\{-a a^{\prime 2}-a+\frac{\Lambda}{3} a^{3}+B\left(\frac{a^{\prime 2}}{a}-\frac{a^{\prime 4}}{6 a}\right)+\frac{B}{2 a}\right\}+F(\eta), \\
& F(\eta)= \pm \sum_{\omega} \ln \left(1 \mp e^{-\omega \eta}\right), \quad \eta=\int d \tau N / a .
\end{aligned}
$$

Here $a^{\prime} \equiv d a / N d \tau$, the first three terms in curly brackets represent the classical Einstein action with a primordial cosmological constant $\Lambda$, the $B$-terms correspond to the contribution of the conformal anomaly and the contribution of the vacuum (Casimir) energy $(B / 2 a)$ of conformal fields on a static Einstein spacetime. $F(\eta)$ is a free energy of these fields - a typical boson or fermion sum over field oscillators with energies $\omega$ on a unit 3-sphere, $\eta$ playing the role of the inverse temperature - an overall circumference of the toroidal instanton measured in units of the conformal time. The constant B,

$$
B=\frac{3 \beta}{4 m_{P}^{2}}=\frac{\beta G}{\pi}
$$


is determined by the coefficient $\beta$ of the topological Gauss-Bonnet invariant $E=R_{\mu \nu \alpha \gamma}^{2}-4 R_{\mu \nu}^{2}+R^{2}$ in the overall conformal anomaly of quantum fields

$$
g_{\mu \nu} \frac{\delta \Gamma_{E}}{\delta g_{\mu \nu}}=\frac{1}{4(4 \pi)^{2}} g^{1 / 2}\left(\alpha \square R+\beta E+\gamma C_{\mu \nu \alpha \beta}^{2}\right),
$$

$\left(C_{\mu \nu \alpha \beta}^{2}\right.$ is the Weyl tensor squared term). For the model of $N_{0}$ scalar, $N_{1 / 2}$ Weyl spinor and $N_{1}$ gauge vector fields it reads [7]

$$
\beta=\frac{1}{360}\left(2 N_{0}+11 N_{1 / 2}+124 N_{1}\right) .
$$

The coefficient $\gamma$ does not contribute to (2.3) because the Weyl tensor vanishes for any FRW metric. The situation with the coefficient $\alpha$ is more complicated. A nonvanishing $\alpha$ induces higher derivative terms $\sim \alpha\left(a^{\prime \prime}\right)^{2}$ in the action and, therefore, adds one extra degree of freedom to the minisuperspace sector of $a$ and $N$ and results in instabilities 1 . But $\alpha$ can be renormalized to zero by adding a finite local counterterm $\sim R^{2}$ admissible by the renormalization theory. We assume this number of degrees of freedom preserving renormalization to keep theory consistent both at the classical and quantum levels [5]. It is interesting that this finite renormalization changes the value of the Casimir energy of conformal fields in closed Einstein cosmology in such a way that for all spins this energy is universally expressed in terms of the same conformal anomaly coefficient $B$ (corresponding to the $B / 2 a$ term in (2.3) 5. 5 . As we will see, this leads to the gravitational screening of the Casimir energy, mediated by the conformal anomaly of quantum fields.

Ultimately, the effective action (2.3) contains only two dimensional constants - the Planck mass squared (or the gravitational constant) $m_{P}^{2}=3 \pi / 4 G$ and the cosmological constant $\Lambda$. They have to be considered as renormalized quantities. Indeed, the effective action of conformal fields contains divergences, the quartic and quadratic ones being absorbed by the renormalization of the initially singular bare cosmological and gravitational constants to yield finite renormalized $m_{P}^{2}$ and $\Lambda$. Logarithmically divergent counterterms have the same structure as curvature invariants in the anomaly (2.6). When integrated over the spacetime closed toroidal FRW instantons they identically vanish because the $\square R$ is a total derivative, Euler number of $S^{3} \times S^{1}$ is zero, $\int d^{4} x g^{1 / 2} E=0$, and $C_{\mu \nu \alpha \beta}=0$. There is however a finite tail of these vanishing logarithmic divergences in the form of the conformal anomaly action which incorporates the coefficient $\beta$ of $E$ in (2.6) and constitutes a major contribution to $\Gamma_{E}$ - the first two $B$-dependent terms of (2.4) 2 . Thus, in fact, this model when considered in the leading order of the $1 / N$-expansion (therefore disregarding loop effects of the graviton and other non-conformal fields) is renormalizable in the minisuperspace sector of the theory.

The path integral (2.2) is dominated by the saddle points - solutions of the equation $\delta \Gamma_{E} / \delta N(\tau)=$ 0 which reads as

$$
\begin{aligned}
& -\frac{a^{\prime 2}}{a^{2}}+\frac{1}{a^{2}}-B\left(\frac{1}{2} \frac{a^{\prime 4}}{a^{4}}-\frac{a^{\prime 2}}{a^{4}}\right)=\frac{\Lambda}{3}+\frac{C}{a^{4}}, \\
& C=\frac{B}{2}+\frac{d F(\eta)}{d \eta}, \quad \eta=2 k \int_{\tau_{-}}^{\tau_{+}} \frac{d \tau}{a} .
\end{aligned}
$$

Note that the usual (Euclidean) Friedmann equation is modified by the anomalous $B$-term and the radiation term $C / a^{4}$. The constant $C$ sets the amount of radiation and satisfies the bootstrap equation (2.9), where $B / 2$ is a contribution of the Casimir energy, and

$$
\frac{d F(\eta)}{d \eta}=\sum_{\omega} \frac{\omega}{e^{\omega \eta} \mp 1}
$$

\footnotetext{
${ }^{1}$ In Einstein theory this sector does not contain physical degrees of freedom at all, which solves the problem of the formal ghost nature of $a$ in the Einstein Lagrangian. Addition of higher derivative term for $a$ does not formally lead to a ghost - the additional degree of freedom has a good sign of the kinetic term as it happens in $f(R)$-theories, but still leads to instabilities discovered in [2].

${ }^{2}$ These terms can be derived from the metric-dependent Riegert action [8] or the action in terms of the conformal factor relating two metrics [9, 10] and generalize the action of [1] to the case of a spatially closed cosmology with $\alpha=0$.
} 
is the energy of the gas of thermally excited particles with the inverse temperature $\eta$. The latter is given in (2.9) by the $k$-fold integral between the turning points of the scale factor history $a(\tau)$, $\dot{a}\left(\tau_{ \pm}\right)=0$. This $k$-fold nature implies that in the periodic solution the scale factor $k$ times oscillates between its maximum and minimum values $a_{ \pm}=a\left(\tau_{ \pm}\right)$.

As shown in [5], such solutions represent the garland-type instantons which exist only in the limited range of the cosmological constant

$$
0<\Lambda_{\min }<\Lambda<\frac{3 \pi}{2 \beta G}
$$

and eliminate the vacuum Hartle-Hawking instantons corresponding to $a_{-}=03$. The period of these quasi-thermal instantons is not a freely specifiable parameter, but as a function of $G$ and $\Lambda$ follows from Eqs. (2.8)-(2.9). Therefore the model does not describe a canonical ensemble, but rather a microcanonical ensemble (see [6]) with only two freely specifiable dimensional parameters - the renormalized gravitational and cosmological constants discussed above.

The upper bound of the range (2.11) is entirely caused by the quantum anomaly - this is a new quantum gravity scale which tends to infinity when one switches the quantum effects off, $\beta \rightarrow 0$. The lower bound $\Lambda_{\min }$ is the effect of both radiation and anomaly, and can be obtained numerically for any field contents of the model. For a large number of conformal fields, and therefore a large $\beta$, the lower bound is of the order $\Lambda_{\min } \sim 1 / \beta G$. Thus the restriction (2.11) can be regarded as a solution of the cosmological constant problem in the early Universe, because specifying a sufficiently high number of conformal fields one can achieve the primordial value of $\Lambda$ well below the Planck scale where the effective theory applies, but high enough to generate sufficiently long inflationary stage. Also this restriction can be potentially considered as a selection criterion for the landscape of string vacua [5, 6].

\section{Cosmological evolution}

The gravitational instantons of the above type can be regarded as setting initial conditions for the cosmological evolution in the physical spacetime with the Lorentzian signature. This can be viewed as nucleation of the Lorentzian spacetime from the Euclidean spacetime at the maximum value of the scale factor $a_{+}=a\left(\tau_{+}\right)$at the turning point of the Euclidean solution $\tau_{+}$- the minimal (zero extrinsic curvature) surface of the instanton. For the contribution of the one-fold instanton to the density matrix of the Universe this nucleation process is depicted in Fig. 1]

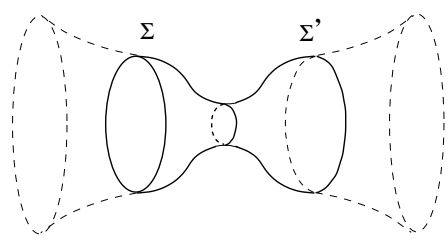

Figure 1: The contribution of the one-fold instanton to the density matrix of the Universe, whose two arguments are associated with with the surfaces $\Sigma$ and $\Sigma^{\prime}$. Dashed lines depict the Lorentzian Universe nucleating at $\Sigma$ and $\Sigma^{\prime}$.

The Lorentzian evolution can be obtained by analytically continuing the Euclidean time into a complex plane by the rule $\tau=\tau_{+}+i t$. Correspondingly the Lorentzian effective equation follows from

\footnotetext{
${ }^{3}$ Hartle-Hawking instantons are ruled out in the statistical sum by their infinite positive effective action which is due to the contribution of the conformal anomaly drastically changing predictions of the tree-level theory.
} 
the Euclidean one (2.8) as

$$
\frac{\dot{a}^{2}}{a^{2}}+\frac{1}{a^{2}}-B\left(\frac{1}{2} \frac{\dot{a}^{4}}{a^{4}}+\frac{\dot{a}^{2}}{a^{4}}\right)=\frac{\Lambda}{3}+\frac{C}{a^{4}},
$$

where the dot from now on denotes the derivative with respect to the Lorentzian time $t$. This can be rewritten in the form

$$
\frac{\dot{a}^{2}}{a^{2}}+\frac{1}{a^{2}}-\frac{B}{2}\left(\frac{\dot{a}^{2}}{a^{2}}+\frac{1}{a^{2}}\right)^{2}=\frac{\Lambda}{3}+\frac{C-B / 2}{a^{4}},
$$

and solved for the Hubble factor as

$$
\begin{aligned}
& \frac{\dot{a}^{2}}{a^{2}}+\frac{1}{a^{2}}=\frac{1}{B}\left\{1-\sqrt{1-2 B\left(\frac{\Lambda}{3}+\frac{\mathcal{C}}{a^{4}}\right)}\right\}, \\
& \mathcal{C} \equiv C-\frac{B}{2} .
\end{aligned}
$$

We have thus obtained a modified Friedmann equation in which the overall energy density, including both the cosmological constant and radiation, very nonlinearly contributes to the square of the Hubble factor.

An interesting property of this equation is that the Casimir energy does not weigh - the term $B / 2 a^{4}$ is completely subtracted from the full radiation density $C / a^{4}$ in the right hand side of (3.2) and under the square root of (3.3). Only "real" thermally excited quanta contribute to the right-hand side of (3.3). Indeed, using (2.9), the radiation contribution $\mathcal{C} / a^{4}$ is seen to read simply as

$$
\frac{\mathcal{C}}{a^{4}}=\frac{1}{a^{4}} \sum_{\omega} \frac{\omega}{e^{\omega \eta} \mp 1} .
$$

This is an example of the gravitational screening which is now being intensively searched for the cosmological constant [11, 12. As we see this mechanism is mediated by the conformal anomaly action, but it applies not to the cosmological constant, but rather to the Casimir energy which has the equation of state of radiation $p=\varepsilon / 3$. This gravitational screening is essentially based on the above mentioned renormalization that eradicates higher derivatives from the effective action and thus preserves the minisuperspace sector free from dynamical degrees of freedom.

After nucleation from the Euclidean instanton at the turning point with $a=a_{+}$and $\dot{a}_{+}=0$ the Lorentzian Universe starts expanding, because

$$
\ddot{a}_{+}=-\left.a \frac{1+\dot{a}^{2}-2 \Lambda a^{2} / 3}{a^{2}-B-B \dot{a}^{2}}\right|_{\tau_{+}}=a_{+} \frac{\sqrt{1-4 C \Lambda / 3}}{a_{+}^{2}-B}>0
$$

(this equation can be derived from (3.3) or obtained by analytic continuation from the Euclidean variational equation $\left.\delta \Gamma_{E}[a, N] / \delta a=0\right)$. Therefore, the radiation quickly dilutes, so that the primordial cosmological constant starts dominating and can generate an inflationary stage. It is natural to assume that the primordial $\Lambda$ is not fundamental, but is due to some inflaton field. This effective $\Lambda$ is nearly constant during the Euclidean stage and the inflation stage, and subsequently leads to a conventional exit from inflation by the slow roll mechanism 4 .

During a sufficiently long inflationary stage, particle production of conformally non-invariant matter takes over the polarization effects of conformal fields. After being thermalized at the exit from inflation this matter gives rise to an energy density $\varepsilon(a)$ which should replace the energy density of

\footnotetext{
${ }^{4}$ In the Euclidean regime this field also stays in the slow roll approximation, but in view of the oscillating nature of a scale factor it does not monotonically decay. Rather it follows these oscillations with much lower amplitude and remains nearly constant during all Euclidean evolution, whatever long this evolution is (as it happens for garland instantons with the number of folds $k \rightarrow \infty$ ).
} 
the primordial cosmological constant and radiation. Therefore, at the end of inflation the combination $\Lambda / 3+\mathcal{C} / a^{4}$ should be replaced according to

$$
\frac{\Lambda}{3}+\frac{\mathcal{C}}{a^{4}} \rightarrow \frac{8 \pi G}{3} \varepsilon(a) \equiv \frac{8 \pi G}{3} \rho(a)+\frac{\mathcal{C}}{a^{4}} .
$$

Here $\varepsilon(a)$ denotes the full energy density including the component $\rho(a)$ resulting from the decay of $\Lambda$ and the radiation density of the primordial conformal matter $\mathcal{C} / a^{4}$. The dependence of $\varepsilon(a)$ on $a$ is of course determined by the equation of state via the stress tensor conservation, and $\rho(a)$ also includes its own radiation component emitted by and staying in (quasi)equilibrium with the barionic part of the full $\varepsilon(a)$.

Thus the modified Friedman equation finally takes the form

$$
\frac{\dot{a}^{2}}{a^{2}}+\frac{1}{a^{2}}=\frac{\pi}{\beta G}\left\{1-\sqrt{1-\frac{16 G^{2}}{3} \beta \varepsilon}\right\},
$$

where we expressed $B$ according to (2.5)

In the limit of small subplanckian energy density $\beta G^{2} \varepsilon \equiv \beta \varepsilon / \varepsilon_{P} \ll 1$ the modified equation goes over into the ordinary Friedmann equation in which the parameter $\beta$ completely drops out

$$
\frac{\dot{a}^{2}}{a^{2}}+\frac{1}{a^{2}}=\frac{8 \pi G}{3} \varepsilon, \quad G^{2} \varepsilon \ll \frac{1}{\beta} .
$$

Therefore within this energy range the standard cosmology is recovered. Depending on the effective equation of state, a wide set of the known scenarios of late cosmological evolution can be obtained, including quintessence [13] and other scenarios of cosmological acceleration [14].

\section{Big boost cosmological acceleration}

The range of applicability of the GR limit (3.9) depends however on $\beta$. This makes possible a very interesting mechanism to happen for a very large $\beta$. Indeed, the value of the argument of the square root in (3.8) can be sufficiently far from 1 even for small $\varepsilon$ provided $\beta \sim N_{\text {cdf }} \gg 1$. Moreover, one can imagine a model with a variable number of the conformal fields $N_{\text {cdf }}(t)$ inducing a time and implicitly a scale factor dependent $\beta, \beta=\beta(a)$. If $\beta(a)$ grows with $a$ faster than the rate of decrease of $\varepsilon(a)$, then the solution of (3.8) can reach the singular point labeled below by $\infty$ at which the square root argument vanishes and the cosmological acceleration becomes infinite. This follows from the expression

$$
\frac{\ddot{a}}{a}=\frac{\pi}{\beta G}\left\{\left(1-\sqrt{1-16 G^{2} \beta \varepsilon / 3}\right)\left(1-\frac{1}{2} \frac{a(G \beta)^{\prime}}{G \beta}\right)+\frac{4}{3} \frac{a\left(G^{2} \beta \varepsilon\right)^{\prime}}{\sqrt{1-16 G^{2} \beta \varepsilon / 3}}\right\},
$$

where prime denotes the derivative with respect to $a$. This expression becomes singular at $t=t_{\infty}$ even though the Hubble factor remains finite when

$$
\begin{aligned}
& \left(G^{2} \beta \varepsilon\right)_{\infty}=\frac{3}{16}, \\
& \left(\frac{\dot{a}^{2}}{a^{2}}+\frac{1}{a^{2}}\right)_{\infty}=\frac{16 \pi}{3}(G \varepsilon)_{\infty} .
\end{aligned}
$$

Assuming for simplicity that the matter density has a dust-like behavior and $\beta$ grows by a power law in $a$

$$
G \varepsilon \sim \frac{1}{a^{3}}, \quad G \beta \sim a^{n}, \quad n>3,
$$


one easily finds an inflection point $t=t_{*}$ when the cosmological acceleration starts after the deceleration stage

$$
\begin{aligned}
& \left(G^{2} \beta \varepsilon\right)_{*}=\frac{3}{4} \frac{n-2}{(n-1)^{2}}, \\
& \left(\frac{\dot{a}^{2}}{a^{2}}+\frac{1}{a^{2}}\right)_{*}=\frac{8 \pi}{3}(G \varepsilon)_{*} \frac{n-1}{n-2} .
\end{aligned}
$$

Of course, for the acceleration stage to start, the Universe should reach this inflection point $t_{*}$ before recollapsing from the point of its maximal expansion. This requirement imposes certain restrictions on the coefficients of the asymptotic behavior of $G \varepsilon$ and $G \beta$, (4.4), which depend on the details of the history of the equation of state for $\varepsilon(a)$ and the dynamics in the number of conformal fields. We will consider these details elsewhere.

Here it is worth only mentioning that the matter density in the vicinity of $t_{*}$ gravitates with a slightly rescaled gravitational constant, Eq.(4.6), while at the singularity the effective gravitational constant doubles, see Eq. (4.3). Also it is useful to comment on the duration of the acceleration stage before reaching the singularity. If we identify our epoch with some instant $t_{0}$ soon after $t_{*}$, then this duration until the singularity can be estimated by disregarding the spatial curvature term. Then it reads as

$$
t_{\infty}-t_{*} \sim \sqrt{B_{0}} \sim H_{0}^{-1},
$$

which is comparable to the age of the Universe. Thus, although the acceleration stage does not pass the eternity test of [15], its duration is very large.

Nevertheless, the evolution ends in this model with the curvature singularity, $\ddot{a} \rightarrow \infty$, reachable in a finite proper time. Unlike the situation with a big brake singularity of [16 it cannot be extended beyond this singularity analytically even by smearing it or taking into account its weak integrable nature. In contrast to [16 the acceleration is positive, which fact allows us to call this singularity a big boost. The effect of the conformal anomaly drives the expansion of the Universe to the maximum value of the Hubble constant (4.3), after which the solution becomes complex. This, of course, does not make the model apriori inconsistent, because for $t \rightarrow t_{*}$ an infinitely growing curvature invalidates the semiclassical and $1 / N$ approximations. This is a new essentially quantum stage which requires the UV completion of the effective low-energy theory.

\section{AdS/CFT and Randall-Sundrum braneworld setup}

What can be the mechanism of a variable and indefinitely growing $\beta$ ? One such mechanism is well known - phase transitions in cosmology between different vacua can give a mass $m$ to an initially massless particle. This results in the loss of conformal invariance of the corresponding particle, which instead of contributing to the vacuum polarization by its own $\beta$ factor starts generating the ColemanWeinberg type potential $\sim m^{4} \ln \left(m^{2} / \mu^{2}\right)$. However this effect is weak and decreases the effective value of $\beta$, which is not what we are after.

Another mechanism was suggested in [6]. It relies on the possible existence, motivated by string theory, of extra dimensions whose size is evolving in time. Indeed, theories with extra dimensions provide a qualitative mechanism to promote $\beta$ to the level of a moduli variable indefinitely growing with the evolving size $L$ of those dimensions. Indeed $\beta$ basically counts the number $N_{\text {cdf }}$ of conformal degrees of freedom, $\beta \sim N_{\text {cdf }}$ (see Eq.(2.10) ). If one considers a string theory in a space time with more than four dimensions, the extra-dimension being compact with typical size $L$, the effective 4-dimensional fields arise as Kaluza-Klein (KK) and winding modes with masses (see e.g. [17])

$$
m_{n, w}^{2}=\frac{n^{2}}{L^{2}}+\frac{w^{2}}{\alpha^{\prime 2}} L^{2}
$$


(where $n$ and $w$ are respectively the KK and winding numbers), which break their conformal symmetry. These modes remain approximately conformally invariant as long as their masses are much smaller than the spacetime curvature, $m_{n, w}^{2} \ll H_{0}^{2} \sim m_{P}^{2} / N_{\text {cdf }}$. Therefore the number of conformally invariant modes changes with $L$. Simple estimates show that the number of pure KK modes $\left(w=0, n \leq N_{\text {cdf }}\right)$ grows with $L$ as $N_{\mathrm{cdf}} \sim\left(m_{P} L\right)^{2 / 3}$, whereas the number of pure winding modes $\left(n=0, w \leq N_{\mathrm{cdf}}\right)$ grows as $L$ decreases as $N_{\text {cdf }} \sim\left(m_{P} \alpha^{\prime} / L\right)^{2 / 3}$. Thus, it is possible to find a growing $\beta$ in both cases with expanding or contracting extra dimensions. In the first case it is the growing tower of superhorizon KK modes which makes the horizon scale $H_{0} \sim m_{P} / \sqrt{N_{\text {cdf }}} \sim m_{P} /\left(m_{P} L\right)^{1 / 3}$ indefinitely decrease with $L \rightarrow \infty$. In the second case it is the tower of superhorizon winding modes which makes this scale decrease with the decreasing $L$ as $H_{0} \sim m_{P}\left(L / m_{P} \alpha^{\prime}\right)^{1 / 3}$. At the qualitative level of this discussion so far, such a scenario is flexible enough to accommodate the present day acceleration scale (though, at the price of fine-tuning an enormous coefficient of expansion or contraction of $L$ ).

However, string (or rather string-inspired) models can offer a more explicit construction of the ideas put forward previously, as well as help addressing various phenomenological questions which arise in their consideration. In particular, one obvious question which arises, should the model considered here be realistic, is what are the possible observable effects of the large number of required conformal fields. Here some guidance can be obtained from the AdS/CFT picture. Indeed, in this picture [18 a higher dimensional theory of gravity, namely type IIB supergravity compactified on $A d S_{5} \times S^{5}$, is seen to be equivalent to a four dimensional conformal theory, namely $\mathcal{N}=4 S U(N) \mathrm{SYM}$, thought to live on the boundary of $A d S_{5}$ space-time. An interesting arena for a slight generalization of these ideas is the Randall-Sundrum model [19] where a 3-brane is put in the inside of $A d S_{5}$ space-time resulting in a large distance recovery of $4 \mathrm{D}$ gravity without the need for compactification. This model has a dual description. On the one hand it can just be considered from a $5 \mathrm{D}$ gravity perspective, on the other hand it can also be described, thanks to the AdS/CFT picture, by a $4 \mathrm{D}$ conformal field theory coupled to gravity.

Indeed, in this picture, the 5D SUGRA - a field-theoretic limit of the type IIB string - induces on the conformal boundary of the underlying AdS background the quantum effective action of the conformally invariant $4 \mathrm{D} \mathcal{N}=4 S U(N)$ SYM theory coupled to the $4 \mathrm{D}$ geometry of the boundary. The multiplets of this CFT contributing according to (2.10) to the total conformal anomaly coefficient $\beta$ are given by $\left(N_{0}, N_{1 / 2}, N_{1}\right)=\left(6 N^{2}, 4 N^{2}, N^{2}\right)$ [21], so that

$$
\beta=\frac{1}{2} N^{2} .
$$

The parameters of the two theories are related by the equation [18, 20, 4]

$$
\frac{L^{3}}{2 G_{5}}=\frac{N^{2}}{\pi},
$$

where $L$ is the radius of the $5 \mathrm{D} A d S$ space-time with the negative cosmological constant $\Lambda_{5}=-6 / L^{2}$ and $G_{5}$ is the $5 \mathrm{D}$ gravitational constant. The radius $L$ is also related to the 't Hooft parameter of the SYM coupling $\lambda=g_{S Y M}^{2} N$ and the string length scale $l_{s}=\sqrt{\alpha^{\prime}}, L=\lambda^{1 / 4} l_{s}$. The generation of the 4D CFT from the local 5D supergravity holds in the limit when both $N$ and $\lambda$ are large. This guarantees the smallness of string corrections and establishes the relation between the weakly coupled tree-level gravity theory in the bulk $\left(G_{5} \rightarrow 0, L \rightarrow \infty\right)$ and the strongly coupled $4 \mathrm{D}$ CFT $\left(g_{S Y M}^{2} \gg 1\right)$. Moreover, as said above, the AdS/CFT correspondence explains the mechanism of recovering general relativity theory on the 4D brane of the Randall-Sundrum model [20, 4]. The 4D gravity theory is induced on the brane from the $5 \mathrm{D}$ theory with the negative cosmological constant $\Lambda_{5}=-6 / L^{2}$. In the one-sided version of this model the brane has a tension $\sigma=3 / 8 \pi G_{5} L$ (the $4 \mathrm{D}$ cosmological constant is given by $\Lambda_{4}=8 \pi G_{4} \sigma$ ), and the $4 \mathrm{D}$ gravitational constant $G_{4} \equiv G$ turns out to be

$$
G=\frac{2 G_{5}}{L} .
$$


One recovers 4D General Relativity at low energies and for distances larger than the radius of the AdS bulk, $L$. Thus, the CFT dual description of the 5D Randall-Sundrum model is very similar to the model considered above. Moreover, even though the CFT effective action is not exactly calculable for $g_{S Y M}^{2} \gg 1$ it is generally believed that its conformal anomaly is protected by extended SUSY [23] and is exactly given by the one-loop result (2.6). Therefore it generates the exact effective action of the anomalous (conformal) degree of freedom given by (2.4), which guarantees a good $1 / N_{\text {cdf }^{-}}$ approximation for the gravitational dynamics.

Applying further the above relations it follows a relation between our $\beta$ coefficient and the radius $L$ of the $A d S$ space-time, given by $\beta G=\pi L^{2} / 2$. Introducing this in the modified Friedmann equation (3.8), the latter becomes explicitly depending on the size of the 5D AdS spacetime as given by

$$
\frac{\dot{a}^{2}}{a^{2}}+\frac{1}{a^{2}}=\frac{2}{L^{2}}\left\{1-\sqrt{1-L^{2}\left(\frac{8 \pi G}{3} \rho+\frac{\mathcal{C}}{a^{4}}\right)}\right\},
$$

where we have reintroduced the decomposition (3.7) of the full matter density into the decay product of the inflationary and matter domination stages $\rho$ and the thermal excitations of the primordial CFT (3.5).

For low energy density, $G L^{2} \rho \ll 1$ and $L^{2} \mathcal{C} / a^{4} \ll 1$, in the approximation beyond the leading order, cf. Eq.(3.9), this equation read:5

$$
\frac{\dot{a}^{2}}{a^{2}}+\frac{1}{a^{2}} \simeq \frac{8 \pi G}{3} \rho\left(1+\frac{2 \pi G L^{2}}{3} \rho\right)+\frac{\mathcal{C}}{a^{4}}
$$

and coincides with the modified Friedmann equation in the Randall-Sundrum model 22$]$

$$
\frac{\dot{a}^{2}}{a^{2}}+\frac{1}{a^{2}}=\frac{8 \pi G}{3} \rho\left(1+\frac{\rho}{2 \sigma}\right)+\frac{\mathcal{C}}{a^{4}},
$$

where $\sigma=3 / 8 \pi G_{5} L=3 / 4 \pi G L^{2}$ is the Randal-Sundrum brane tension and $\mathcal{C}$ is the braneworld constant of motion [22, 25]. Note that the thermal radiation on the brane (of non-Casimir energy nature) is equivalent to the mass of the bulk black hole associated with this constant. This fact can be regarded as another manifestation of the AdS/CFT correspondence in view of the known duality between the bulk black hole and the thermal CFT on the brane [25. Thus indeed anomaly driven cosmology coincides with the Randall-Sundrum one in the limit of low density of matter and radiation.

Interestingly, this comparison between our model and the Randall-Sundrum framework also allows one to have some insight on the phenomenologically allowed physical scales. Indeed, it is well known that the presence of an extra-dimension in the Randall-Sundrum model, or in the dual language, that of the CFT, manifests itself typically at distances lower that the $A d S$ radius $L$. Hence, it is perfectly possible to have a large number of conformal fields in the Universe, à la Randall-Sundrum, without noticing their presence in the everyday experiments, provided $L$ is small enough. Moreover, if one uses the scenario of [5] to set the initial conditions for inflation, it provides an interesting connection between the Hubble radius of inflation, given by eq. (2.11), and the distance at which the presence of the CFT would manifest itself in gravity experiments, both being given by $L$. Last, it seems natural in a string theory setting, to imagine that the $A d S$ radius $L$ can depend on time, and hence on the scale factor.

In this case, assuming that the AdS/CFT picture still holds when $L$ is adiabatically evolving, one can consider the possibility that $G L^{2} \varepsilon$ is large, and that $L^{2}(t)$ grows faster than $G \varepsilon(t)$ decreases during the cosmological expansion. One would then get the cosmological acceleration scenario of the above type followed by the big boost singularity.

In this case, however, should this acceleration scenario correspond to the present day accelerated expansion, $L$ should be of the order of the present size of the Universe, i.e. $L^{-2} \sim H_{0}^{2}$. Since the

\footnotetext{
${ }^{5}$ We assume that the dark radiation term is redshifted for growing $a$ faster than matter term and expand to the second order in $\rho$, but the first order in $\mathcal{C}$.
} 
Randall-Sundrum mechanism recovers 4D GR only at distances beyond the curvature radius of the AdS bulk, $r \gg L$, it means that local gravitational physics of our model (5.5) at the acceleration stage is very different from the $4 \mathrm{D}$ general relativity. Thus this mechanism can hardly be a good candidate for generating dark energy in real cosmology.

\section{Anomaly driven cosmology and the DGP model}

It is interesting that there exists an even more striking example of a braneworld setup dual to our anomaly driven model. This is the generalized DGP model 24] including together with the 4D and 5D Einstein-Hilbert terms also the 5D cosmological constant, $\Lambda_{5}$, in the special case of the vacuum state on the brane with a vanishing matter density $\rho=0$. In contrast to the Randall-Sundrum model, for which this duality holds only in the low energy limit - small $\rho$ and small $\mathcal{C} / a^{4}$, vacuum DGP cosmology exactly corresponds to the model of [5] with the $4 \mathrm{D}$ cosmological constant $\Lambda$ simulated by the $5 \mathrm{D}$ cosmological constant $\Lambda_{5}$.

Indeed, in this model (provided one neglects the bulk curvature), gravity interpolates between a $4 \mathrm{D}$ behaviour at small distances and a $5 \mathrm{D}$ behaviour at large distances, with the crossover scale between the two regimes being given by $r_{c}$,

$$
\frac{G_{5}}{2 G}=r_{c},
$$

and in the absence of stress-energy exchange between the brane and the bulk, the modified Friedmann equation takes the form [26]

$$
\frac{\dot{a}^{2}}{a^{2}}+\frac{1}{a^{2}}-r_{c}^{2}\left(\frac{\dot{a}^{2}}{a^{2}}+\frac{1}{a^{2}}-\frac{8 \pi G}{3} \rho\right)^{2}=\frac{\Lambda_{5}}{6}+\frac{\mathcal{C}}{a^{4}} .
$$

Here $\mathcal{C}$ is the same as above constant of integration of the bulk Einstein's equation, which corresponds to a nonvanishing Weyl tensor in the bulk (or a mass for a Schwarzschild geometry in the bulk) [22, 25]. It is remarkable that this equation with $\rho=0$ exactly coincides with the modified Friedmann equation of the anomaly driven cosmology (3.2) under the identifications

$$
\begin{aligned}
& B \equiv \frac{\beta G}{\pi}=2 r_{c}^{2}, \\
& \Lambda=\frac{\Lambda_{5}}{2} .
\end{aligned}
$$

These identifications imply that in the DGP limit $G \ll r_{c}^{2}$, the anomaly coefficient $\beta$ is much larger than 1.

This looks very much like the generation of the vacuum DGP model for any value of the dark radiation $\mathcal{C} / a^{4}$ from the anomaly driven cosmology with a very large $\beta \sim m_{P}^{2} r_{c}^{2} \gg 1$. However, there are several differences. A first important difference between the conventional DGP model and the anomaly driven DGP is that the former does not incorporate the self-accelerating branch [26, 27, of the latter. This corresponds to the fact that only one sign of the square root is admissible in Eq.(3.3) - a property dictated by the instanton initial conditions at the nucleation of the Lorentzian spacetime from the Euclidean one (see Eq.(3.6) ). So, one does not have to worry about possible instabilities associated with the self-accelerating branch $[28$.

Another important difference concerns the way the matter energy density manifests itself in the Friedmann equation for the non-vacuum case. In our 4D anomaly driven model it enters the right hand side of the equation as a result of the decay (3.7) of the effective $4 \mathrm{D}$ cosmological constant $\Lambda$, while in the DGP model it appears inside the parenthesis of the left hand side of equation (6.2). Therefore, the DGP Hubble factor reads as

$$
\frac{\dot{a}^{2}}{a^{2}}+\frac{1}{a^{2}}=\frac{8 \pi G}{3} \rho+\frac{1}{2 r_{c}^{2}}\left\{1-\sqrt{1-4 r_{c}^{2}\left(\frac{\Lambda_{5}}{6}+\frac{\mathcal{C}}{a^{4}}-\frac{8 \pi G}{3} \rho\right)}\right\}
$$


(note the negative sign of $\rho$ under the square root and the extra first term on the right hand side) and in the limit of small $\rho, \mathcal{C} / a^{4}$ and $\Lambda_{5}$ yields the behavior very different from the GR limit of the anomaly driven model (3.9),

$$
\frac{\dot{a}^{2}}{a^{2}}+\frac{1}{a^{2}} \simeq \frac{\Lambda_{5}}{6}+\frac{\mathcal{C}}{a^{4}}+r_{c}^{2}\left(\frac{\Lambda_{5}}{6}+\frac{\mathcal{C}}{a^{4}}-\frac{8 \pi G}{3} \rho\right)^{2} .
$$

For vanishing $\Lambda_{5}$ and $\mathcal{C} / a^{4}$ this behavior corresponds to the $5 \mathrm{D}$ dynamical phase [26, 27] which is realized in the DGP model for a very small matter energy density on the brane $\rho \ll 3 / 32 \pi G r_{c}^{2} \sim$ $m_{P}^{2} / r_{c}^{2}$.

Of course, in this range the DGP braneworld reduces to a vacuum brane, but one can also imagine that the 5D cosmological constant decays into matter constituents similar to (3.7) and thus simulates the effect of $\rho$ in Eq. (3.8). This can perhaps provide us with a closer correspondence between the anomaly driven cosmology and the non-vacuum DGP case. But here we would prefer to postpone discussions of such scenarios to future analyses and, instead, focus on the generalized single-branch DGP model to show that it also admits the cosmological acceleration epoch followed by the big boost singularity.

Indeed, for positive $\Lambda_{5}$ satisfying a very weak bound

$$
\Lambda_{5}>\frac{3}{2 r_{c}^{2}}
$$

Eq. (6.5) has a solution for which, during the cosmological expansion with $\rho \rightarrow 0$, the argument of the square root vanishes and the acceleration tends to infinity (prime again denotes the derivative with respect to $a$ )

$$
\frac{\ddot{a}}{a} \simeq \frac{\left[r_{c}^{2}\left(\frac{\Lambda_{5}}{6}+\frac{\mathcal{C}}{a^{4}}-\frac{8 \pi G}{3} \rho\right)\right]^{\prime}}{r_{c}^{2} \sqrt{1-4 r_{c}^{2}\left(\frac{\Lambda_{5}}{6}+\frac{\mathcal{C}}{a^{4}}-\frac{8 \pi G}{3} \rho\right)}} \rightarrow \pm \infty .
$$

This is the big boost singularity labeled similarly to (4.3) by $\infty$ and having a finite Hubble factor

$$
\begin{aligned}
& \left(\frac{\Lambda_{5}}{6}+\frac{\mathcal{C}}{a^{4}}-\frac{8 \pi G}{3} \rho\right)_{\infty}=\frac{1}{4 r_{c}^{2}}, \\
& \left(\frac{\dot{a}^{2}}{a^{2}}+\frac{1}{a^{2}}\right)_{\infty}=\frac{\Lambda_{5}}{6}+\frac{1}{4 r_{c}^{2}} .
\end{aligned}
$$

For the effective $a$-dependence of $r_{c}^{2}$ and $G \rho$ analogous to (4.4), $r_{c}^{2}(a) \sim a^{n}$ and $G \rho(a) \sim 1 / a^{3}$, the acceleration becomes positive at least for $n \geq 0$,

$$
\frac{\ddot{a}}{a} \simeq \frac{n+32 \pi G r_{c}^{2} \rho}{4 r_{c}^{2} \sqrt{1+4 r_{c}^{2}\left(\frac{8 \pi G}{3} \rho-\frac{\Lambda_{5}}{6}-\frac{\mathcal{C}}{a^{4}}\right)}} \rightarrow+\infty .
$$

Thus, the single-branch DGP cosmology can also lead to a big boost version of acceleration. For that to happen, one does not actually need a growing $r_{c}$ (which can be achieved at the price of having a time dependent $G_{5}$ - itself some kind of a modulus, in a string inspired picture). The DGP crossover scale $r_{c}$ can be constant, $n=0$, and the big boost singularity will still occur provided the lower bound (6.7) is satisfied 6 . When $\Lambda_{5}$ violates this bound, the acceleration stage is eternal with the asymptotic value of the Hubble factor squared $\dot{a}^{2} / a^{2}=\left(1-\sqrt{1-2 r_{c}^{2} \Lambda_{5} / 3}\right) / 2 r_{c}^{2}$.

\footnotetext{
${ }^{6}$ Or, more precisely, its small modification due to the dark radiation contribution $\mathcal{C} / a^{4}$ which is very small at late stages of expansion.
} 


\section{Conclusions}

To summarize, we have obtained the modified Friedmann equation in the anomaly driven cosmology with the microcanonical density matrix initial conditions suggested in [5, 6]. This equation exhibits a gravitational screening of the quantum Casimir energy of conformal fields - this part of the total energy density does not weigh, being degravitated due to the contribution of the conformal anomaly. Also, in the low-density limit this equation does not only recover the general relativistic behavior, but also establishes a good correspondence with the dynamics of the Randall-Sundrum cosmology via the AdS/CFT duality. Moreover, for very large and rapidly growing value of the Gauss-Bonnet coefficient $\beta$ in the conformal anomaly this equation features a regime of cosmological acceleration followed by a big boost singularity. At this singularity the scale factor acceleration grows in finite proper time up to infinity with a finite limiting value of the Hubble factor, when the Universe again enters a quantum phase demanding for its description an UV completion of the low-energy semiclassical theory.

A natural mechanism of growing $\beta$ can be based on the idea of adiabatically evolving scale associated with extra dimensions [6] and realized within the picture of AdS/CFT duality, according to which the conformal field theory is induced on the 4D brane from the $5 \mathrm{D}$ non-conformal theory in the bulk. As is well known, this duality underlies the justification of the $4 \mathrm{D}$ general relativistic limit in the Randall-Sundrum model 20, 4. Here we observed an extended status of this duality from the cosmological perspective - the generalized Randall Sundrum model with the Sschwarzschild-AdS bulk was shown to be equivalent to the anomaly driven cosmology for small energy density. In particular, the radiation contents of the latter was shown to be equivalent to the dark radiation term $\mathcal{C} / a^{4}$ pertinent to the Randall-Sundrum braneworld with a bulk black hole of mass $\mathcal{C}$ (well-known duality of the bulk black hole and the thermal CFT on the brane [25]).

It is interesting that the initial conditions of anomaly driven model establish the relation between the amount of radiation $\mathcal{C}$ and the product of renormalized cosmological and gravitational constants $G \Lambda \sim \Lambda / m_{P}^{2}$ - the corollary of the closed system of equations (2.8)-(2.9) [5]. Such a relation is not known in the standard $S U(N)$ AdS/CFT version of the Randall-Sundrum scenario valid for the effective $\Lambda=0$ and large $N^{2} \sim N_{\text {cdf }} \sim \beta \gg 1$. But this is consistent with the fact that the solution of the bootstrap equations (2.8)-(2.9) has a scaling behavior in $N_{\text {cdf }} \sim N^{2}, \Lambda \rightarrow \Lambda / N_{\text {cdf }}, \mathcal{C} \rightarrow N_{\text {cdf }} \mathcal{C}$ [5], which simply implies the limit of $N \rightarrow \infty$ in this scenario. This limit justifies the semiclassical approximation applicable in accordance with (2.11) in the range of curvature much below the Planck scale.

Another intriguing observation concerns establishing the exact correspondence between the anomaly driven cosmology and the vacuum DGP model generalized to the case of a nonvanishing $\Lambda_{5}$. In this case a large $\beta$ is responsible for the large crossover scale $r_{c}$, (6.1). For positive $\Lambda_{5}$ satisfying the lower bound (6.7) this model also features a big boost scenario even for stabilized $\beta$. Below this bound (but still for positive $\Lambda_{5}>0$, because a negative $\Lambda_{5}$ would imply the point of maximal expansion from which the Universe starts recollapsing) the cosmological evolution eventually enters eternal acceleration scenario. However, the DGP model with a matter on the brane can hardly be equivalent to the $4 \mathrm{D}$ anomaly driven cosmology, unless one has some mechanism of decaying $\Lambda_{5}$ and simulating matter density on the brane.

Unfortunately, the AdS/CFT correspondence with adiabatically evolving scale of extra dimension cannot incorporate the phenomenology of the observable dark energy, because the local gravitational physics of this model becomes very different from the $4 \mathrm{D}$ general relativity. Thus, macabre perspective of being ripped by infinite tidal forces at Big Boost singularity seems being postponed. AdS/CFTcorrespondence - a perfect nonperturbative tool in mathematical physics — still remains a thing in itself from the perspective of applied astroparticle cosmology.

In general, the idea of a very large central charge of CFT algebra, underlying the solution of the hierarchy problem in the dark energy phenomenon and particle phenomenology, seems hovering in current literature 29, 30. Our idea of a big growing $\beta$ belongs to the same scope, but its realization seems escaping phenomenological framework. Probably some other modification of this idea can be 
more productive. In particular, another qualitative mechanism of running $\beta$ could be based on the field-theoretic implementation of winding modes. These modes do not seem to play essential role in the AdS/CFT picture with a big scale of extra dimensions $L$, because they are heavy in the big $L$ limit. On the contrary, this mechanism is expected to work in the opposite case of contracting extra dimensions, for which the restrictions imposed by local gravitational physics do not seem to apply (as long as for $L \rightarrow 0$ the short-distance correction go deeper and deeper into UV domain). We hope to consider the mechanism of winding modes in accelerating cosmology elsewhere.

\section{Acknowledgements}

A.B. is grateful for hospitality of the Laboratory APC, CNRS, University Paris 7, Paris, where a major part of this work has been done. His work was also supported by the Russian Foundation for Basic Research under the grant No 05-01-00996 and the grant LSS-4401.2006.2. A.Yu.K. was supported by the RFBR grant 05-02-17450 and the grant LSS-1157.2006.2. A.B. and C.D. wish to thank G.Gababadze and R.Woodard for discussions.

\section{References}

[1] M.V.Fischetti, J.B.Hartle and B.L.Hu, Phys. Rev. D 20, 1757 (1979).

[2] A.A.Starobinsky, Phys. Lett. 91B, 99 (1980).

[3] J.B.Hartle and S.W.Hawking, Phys.Rev. D 28 (1983) 2960.

[4] S.W. Hawking, T. Hertog and H.S. Reall, Phys.Rev. D62 (2000) 043501.

[5] A.O.Barvinsky and A.Yu.Kamenshchik, J. Cosmol. Astropart. Phys. 09, 014 (2006), hep-th/0605132 Phys. Rev. D74, 121502 (2006), hep-th/0611206

[6] A.O.Barvinsky, Phys. Rev. Lett. 99 (2007) 071301, hep-th/0704.0083

[7] M.J.Duff, Class. Quant. Grav 11, 1387 (1994), hep-th/9308075.

[8] R.J.Riegert, Phys. Lett. 134 B, 56 (1984); P.O.Mazur and E.Mottola, Phys. Rev. D 64, 104022 (2001).

[9] E.S.Fradkin and A.A.Tseytlin, Phys. Lett. 134 B, 187 (1984).

[10] A.O.Barvinsky, A.G.Mirzabekian and V.V.Zhytnikov, "Conformal decomposition of the effective action and covariant curvature expansion", gr-qc/9510037.

[11] N.C.Tsamis and R.P.Woodard, Nucl. Phys. B 474, 235 (1996).

[12] G.Dvali, S.Hofmann and J.Khoury, "Degravitation of the Cosmological Constant and Graviton Width", hep-th/0703027.

[13] R.R. Caldwell, R. Dave and P.J. Steinhardt, Phys. Rev. Lett. 80, 1582-1585 (1998); L.-M. Wang, R.R. Caldwell, J.P. Ostriker and P.J. Steinhardt, Astrophys. J. 530, 17 (2000).

[14] A.Yu. Kamenshchik, U. Moschella and V. Pasquier, Phys. Lett. B511, 265 (2001); V. Sahni and A.A. Starobinsky, Int. J. Mod. Phys. D9, 373 (2000); D15, 2105 (2006).

[15] A.M.Polyakov, "De Sitter Space and Eternity", hep-th/0709.2899.

[16] V. Gorini, A.Yu. Kamenshchik, U. Moschella and V. Pasquier, Phys. Rev. D69, 123512 (2004). 
[17] J.Polchinski, String Theory (Cambridge University Press, Cambridge, 1998).

[18] J.Maldacena, Adv. Theor. Math. Phys. 2231 (1998); Int. J. Theor.Phys. 381113 (1999), hep-th/9711200 E.Witten, Adv. Theor. Math. Phys. 2, 253 (1998); S.S.Gubser, I.R.Klebanov and A.M.Polyakov, Phys. Lett. B428, 105 (1998), hep-th/9802109.

[19] L. Randall and R. Sundrum, Phys. Rev. Lett. 83 (1999) 4690 arXiv:hep-th/9906064.

[20] S.Gubser, Phys. Rev. D63, 084017 (2001), hep-th/9912001.

[21] M.J.Duff and J.T.Liu, Phys. Rev. Lett. 85, 2052 (2000), hep-th/0003237.

[22] P.Binetruy, C.Deffayet and D.Langlois, Phys. Lett. 477275 (2000), hep-th/9910219.P. Binetruy, C. Deffayet, U. Ellwanger and D. Langlois, Phys. Lett. B 477 (2000) 285 arXiv:hep-th/9910219.

[23] Hong Liu and A.A. Tseytlin, Nucl.Phys. B533, 88 (1998), hep-th/9804083

[24] G. R. Dvali, G. Gabadadze and M. Porrati, Phys. Lett. B 485 (2000) 208 arXiv:hep-th/0005016.

[25] T. Shiromizu, K. i. Maeda and M. Sasaki, Phys. Rev. D 62, 024012 (2000) arXiv:gr-qc/9910076. P. Kraus, JHEP 9912, 011 (1999) arXiv:hep-th/9910149.

[26] C.Deffayet, Phys. Lett. B 502, 199 (2001), hep-th/0010186.

[27] C.Deffayet, G.Dvali and G.Gabadadze, Phys. Rev. D 65, 044023 (2002), astro-ph/0105068.

[28] M. A. Luty, M. Porrati and R. Rattazzi, JHEP 0309 (2003) 029 arXiv:hep-th/0303116. A. Nicolis and R. Rattazzi, JHEP 0406 (2004) 059 arXiv:hep-th/0404159. G. Dvali, New J. Phys. 8 (2006) 326 arXiv:hep-th/0610013. K. Koyama, Phys. Rev. D 72 (2005) 123511 arXiv:hep-th/0503191]. D. Gorbunov, K. Koyama and S. Sibiryakov, Phys. Rev. D 73 (2006) 044016 [arXiv:hep-th/0512097]. C. Charmousis, R. Gregory, N. Kaloper and A. Padilla, JHEP 0610 (2006) 066 [arXiv:hep-th/0604086]. C. Deffayet, G. Gabadadze and A. Iglesias, JCAP 0608 (2006) 012 arXiv:hep-th/0607099. K. Izumi, K. Koyama, O. Pujolas and T. Tanaka, Phys. Rev. D 76, 104041 (2007) arXiv:0706.1980 [hep-th]]. R. Gregory, N. Kaloper, R. C. Myers and A. Padilla, JHEP 0710 (2007) 069 arXiv:0707.2666 [hep-th]].

[29] N.Arkani-Hamed, S.Dimopoulos, G.Dvali and G.Gabadadze, "Non-Local Modification of Gravity and the Cosmological Constant Problem", hep-th/0209227.

[30] G.Dvali, "Black Holes and Large N Species Solution to the Hierarchy Problem", arXiv:0706.2050, G.Dvali and M.Redi, "Black Hole Bound on the Number of Species and Quantum Gravity at LHC", arXiv:0710.4344 [hep-th]. 